\title{
ISOLATED GALAXIES, PAIRS AND GROUPS OF GALAXIES
}

\author{
I. Kuneva and M. Kalinkov \\ Department of Astronomy, Bulgarian Academy of Sciences \\ 72 Lenin blvd, 1184 Sofia, Bulgaria
}

We searched for isolated galaxies, pairs and groups of galaxies in the CfA survey (Huchra et al. 1983). It was assumed that the distances to galaxies are given by $\mathrm{R}=$ $\mathrm{V} / \mathrm{H}_{0}$, where $\mathrm{H}_{0}=100 \mathrm{~km} \mathrm{~s}^{-1} \mathrm{Mpc}^{-1}$ and $\mathrm{R}>6 \mathrm{Mpc}$.

The searching procedure is close to those, applied to find superclusters of galaxies (Kalinkov and Kuneva 1985,1986). A sphere with fixed radius $\mathrm{r}^{*}$ is described around each galaxy. The mean spatial density in the sphere is $m$. Let $G^{1}$ be any galaxy and $G^{2}$ be its nearest neighbor at a distance $R_{2}$. If $R_{2}$ exceeds the $95 \%$ quintile in the distribution of the distances of the second neighbors, then $\mathrm{G}^{1}$ is an isolated galaxy. Let the midpoint of $G^{1}$ and $G^{2}$ be $\mathrm{O}_{2}$ and $r_{2}=R_{2} / 2$. For the volume $V_{2}$, defined with the radius $r_{2}$, the density $\mathrm{D}_{2}=2 / \mathrm{V}_{2}$ is calculated. Here 2 stands for the number of galaxies in the volume $\mathrm{V}_{2}$. We use inequalities to establish regions with density enhancements higher than $\mathrm{k} u \mathrm{~m}$. Thus, if $D_{2}<k \mu$, the galaxy $G^{2}$ is a single one and the procedure for searching for pairs and groups, beginning with this object is over and we have to pass to another object. If $\mathrm{D}_{2}>\mathrm{k} \mu$, the searching procedure goes on. Let then $\mathrm{G}^{3}$ be the third neighbor of $\mathrm{O}_{2}$ and let $\mathrm{O}_{3}$ be the center of $\mathrm{G}^{1}, \mathrm{G}^{2}$ and $\mathrm{G}^{3} ; \mathrm{r}_{3}=\max \left(\mathrm{O}_{3} \mathrm{G}^{1}, \mathrm{O}_{3} \mathrm{G}^{2}, \mathrm{O}_{3} \mathrm{G}^{3}\right)$ is calculated and around $\mathrm{O}_{3}$ we describe a sphere with radius $r_{3}$ of volume $V_{3}$ and density $D_{3}=3 / V_{3}$. If $D_{3}<k \mu$, it might be concluded that the galaxies $G^{1}$ and $G^{2}$ form a pair for the specific choice of $\mathrm{k}$ and $\mathrm{r}$. The pair is isolated, if the distance $\mathrm{O}_{2} \mathrm{G}^{3}$ exceeds the $95 \%$ quintile in the distribution of the distances of the third neighbors. Then the procedure for the galaxy $\mathrm{G}^{1}$ ends. For $\mathrm{D}_{3}>\mathrm{k} \mu$, the procedure continues. Generally, the procedure for searching for multiplets continues for $\mathrm{D}_{\mathrm{i}}>\mathrm{k} \mu$. If $\mathrm{D}<\mathrm{k} \mu$, the procedure stops with the finding of a multiplet consisting of $\mathrm{i}-1$ members $(\mathrm{i}=2,3, \ldots)$ and the test for isolation with the $95 \%$ quintile in the distribution of the distances to the $\mathrm{i}$-th neighbors.

The algorithm is objective, but it is not always commutative because of the local character of the spatial density. The main difference however between our and some other procedures is the local density. In many cases our groups are closed - it means that the multiplets are independent of the choice of galaxy with which the search has started. But there are some groups which are open - their membership is slightly different, depending on the galaxy with which the procedure has started.

Here the authors present the groups - isolated and nonisolated - with $n>3$, found in the CfA survey in the Northern galactic hemisphere. The parameters used are $\mathrm{k}=10$ and $\mathrm{r}^{*}=5 \mathrm{Mpc}$. Table 1 contains: (1) the group number, (2) the galaxy, nearest to the multiplet center, (3) multiplicity $n,(4)$ the brightest galaxy if it is not listed in (2); (5) and (6) are R.A. and Dec. (1950), (7) - mean distance D in Mpc. Further there are the mean density $\rho(8)$ of the multiplet (galaxies $\mathrm{Mpc}^{-3}$ ), (9) the density $\rho^{*}$ for $\mathrm{r}^{*}=5 \mathrm{Mpc}$ and (10) the density $\rho_{g}$ for the group with its nearest neighbor. The parenthesized digits for densities in the last three columns are powers of ten. 
TABLE 1

\begin{tabular}{|c|c|c|c|c|c|c|c|c|c|}
\hline & Center & $n$ & Brightest & $\alpha$ & $\delta$ & $\mathrm{D}$ & $\rho$ & $\rho^{*}$ & \\
\hline 1 & N2769 & 4 & N2693 & 902.0 & 5109 & 48.5 & $5.8(-1)$ & $1.3(-2)$ & $6.8(-2)$ \\
\hline 2 & U04906 & 3 & N2701 & 905.8 & 5253 & 22.8 & $6.7(-1)$ & $1.7(-2)$ & $4.0(-2)$ \\
\hline 3 & $\mathrm{~N} 2780$ & 3 & $\mathrm{~N} 2770$ & 908.4 & 3433 & 19.7 & 6.1 & $2.9(-2)$ & $5.4(-2)$ \\
\hline 4 & N2799 & 4 & $\mathrm{~N} 2712$ & & 4229 & 18.2 & $4.7(-1)$ & $4.4(-2)$ & $.4(-2)$ \\
\hline 5 & U04870 & 3 & & 914.4 & 4641 & 42.7 & $3.2(-1)$ & $9.6(-3)$ & $2.4(-2)$ \\
\hline 6 & N2856 & 4 & N2776 & 917.5 & 4712 & 26.7 & $4.8(-1)$ & $1.1(-2)$ & $4.6(-2)$ \\
\hline 7 & N2814 & 4 & $\mathrm{~N} 2805$ & 919.2 & 6400 & 16.4 & $9.5(-1)$ & $8.8(-2)$ & $9.9(-2)$ \\
\hline 8 & N2914 & 4 & U05189 & 933.5 & 1002 & 31.7 & $8.2(-1)$ & $1.1(-2)$ & $2.2(-2)$ \\
\hline 9 & N3024 & 3 & N3020 & 949.1 & 1151 & 14.8 & 2.6 & $8.6(-2)$ & $3.0(-1)$ \\
\hline 10 & N3011 & 7 & N3003 & 949.3 & 3202 & 14.9 & 1.7 & $1.2(-1)$ & $5.6(-1)$ \\
\hline 11 & U05520 & 3 & N2909 & 1002.9 & 6540 & 33.1 & 2.7(-1) & $1.3(-2)$ & $4.3(-2)$ \\
\hline 12 & U05459 & 5 & N3079 & 1004.9 & 5453 & 11.1 & 2.9 & $2.2(-1)$ & $6.0(-1)$ \\
\hline 13 & I 591 & 3 & N3107 & 1005.6 & 1306 & 27.5 & 1.1 & $2.1(-2)$ & $2.8(-2)$ \\
\hline 14 & N3104 & 4 & N3184 & 1009.3 & 4356 & 6.1 & 4.2 & $3.7(-1)$ & $7.6(-1)$ \\
\hline 15 & N3169 & 5 & N3166 & 1011.1 & 420 & 13.0 & 1.5 & $1.4(-1)$ & $4.9(-1)$ \\
\hline 16 & N3189 & 10 & N3227 & 1019.6 & 2203 & 13.0 & 1.8 & $1.7(-1)$ & 1.2 \\
\hline 17 & N3245 & 5 & & 1025.5 & 2824 & 13.9 & 1.9 & $1.6(-1)$ & $6.5(-1)$ \\
\hline 18 & N3353 & 3 & N3310 & 1035.7 & 5526 & 9.6 & $1.0(+1)$ & $2.9(-1)$ & $3.3(-1)$ \\
\hline 19 & U05760 & 7 & N3367 & 10 & 1400 & 29.9 & $3.4(-1)$ & $2.7(-2)$ & $7.4(-2)$ \\
\hline 20 & N3346 & 5 & N3338 & & 1438 & 12.6 & $2.2 \cdot$ & $1.8(-1)$ & $6.7(-1)$ \\
\hline 21 & N3381 & 6 & N3294 & 1042.4 & 3438 & 15.9 & 1.4 & $1.1(-1)$ & $4.1(-1)$ \\
\hline 22 & U05955 & 3 & N3403 & 1043.0 & 7320 & 11.9 & 3.5 & $1.6(-1)$ & $1.2(-1)$ \\
\hline 23 & N3368 & 3 & & 1045.9 & 1252 & 9.0 & $2.0(+1)$ & $3.5(-1)$ & $6.0(-1)$ \\
\hline 24 & N3433 & 3 & & 1050.1 & 1022 & 26.9 & $5.1(-1)$ & $2.5(-2)$ & $3.7(-2)$ \\
\hline 25 & N3414 & 3 & & 1052.6 & 2826 & 14.2 & 5.7 & $1.6(-1)$ & $6.0(-1)$ \\
\hline 26 & N3454 & 5 & N3457 & 1053.2 & 1816 & 11.3 & $1.5(+1)$ & $2.9(-1)$ & 1.3 \\
\hline 27 & N3486 & 3 & N3344 & 1053.2 & 2752 & 7.0 & 6.5 & 4.6(-1) & 1.0 \\
\hline 28 & U06106 & 3 & U06135 & 1059.5 & 4555 & 64.9 & $3.2(-1)$ & $1.2(-2)$ & $1.8(-2)$ \\
\hline 29 & N3489 & 5 & N3627 & & 1332 & 7.1 & 6.1 & $4.3(-1)$ & 1.7 \\
\hline 30 & N36 & 9 & N3G & & 5757 & 19.7 & 1.4 & $5.3(-2)$ & $3.8(-1)$ \\
\hline 31 & U06296 & 3 & N3507 & & 1816 & 9.7 & $1.1(+1)$ & $4.7(-1)$ & 1.6 \\
\hline 32 & N35 & 4 & & 111 & 5241 & 28.6 & $2.1(-1)$ & $2.1(-2)$ & $7.4(-2)$ \\
\hline 33 & N3628 & 3 & & 1115.6 & 1512 & 8.3 & 5.4 & $4.4(-1)$ & $8.5(-1)$ \\
\hline 34 & U06345 & 3 & N3630 & 1116.8 & 337 & 15.8 & 2.3 & $7.3(-2)$ & $1.7(-1)$ \\
\hline 35 & N3686 & 5 & N3596 & 1120.1 & 1705 & 11.9 & 7.6 & $4.1(-1)$ & 1.7 \\
\hline 36 & N3665 & 4 & & 1120.8 & 3901 & 20.8 & 8.6 & $3.2(-2)$ & $1.3(-1)$ \\
\hline 37 & N3556 & 3 & & 1129.1 & 5514 & 6.8 & 5.7 & 3.4-(1) & 1.2 \\
\hline 38 & N3788 & 4 & N3687 & 1132.6 & 3146 & 26.2 & $2.3(-1)$ & $2.1(-2)$ & $6.6(-2)$ \\
\hline 39 & N3755 & 3 & N3813 & 1135.3 & 3622 & 15.4 & 1.3 & $7.4(-2)$ & $4.8(-2)$ \\
\hline
\end{tabular}


TABLE 1 (continued)

\begin{tabular}{|c|c|c|c|c|c|c|c|c|c|}
\hline & Center & $n$ & Brightest & $\alpha$ & $\delta$ & $\mathrm{D}$ & $\rho$ & $\rho^{*}$ & $\rho_{g}$ \\
\hline 40 & N3735 & 3 & & 1135.4 & 7053 & 27.4 & 1.0 & $3.8(-2)$ & $1.1(-1)$ \\
\hline 11 & N3853 & 6 & N3800 & 1138.2 & 1713 & 33.0 & 3.5 & $3.2(-2)$ & $1.8(-1)$ \\
\hline 42 & N3770 & 10 & N3963 & 1138.7 & 5947 & 32.0 & $5.1(-1)$ & $3.6(-2)$ & $1.9(-1)$ \\
\hline 43 & N3817 & 4 & N3839 & 1139.2 & 1036 & 61.0 & $2.0(-1)$ & $1.9(-2)$ & $9.4(-2)$ \\
\hline 44 & N3816 & 3 & & 1140.6 & 2020 & 55.1 & $9.2(-1)$ & $1.1(-2)$ & $4.6(-2)$ \\
\hline 45 & U06575 & 16 & N3898 & 1141.0 & 5857 & 12.3 & 2.6 & $2.2(-1)$ & 1.7 \\
\hline 46 & N3837 & 3 & N3842 & 1141.7 & 2010 & 62.8 & $3.3(-1)$ & $2.9(-2)$ & $1.5(-1)$ \\
\hline 47 & N3900 & 3 & & 1144.0 & 2735 & 18.0 & 3.7 & $6.1(-2)$ & $4.5(-2)$ \\
\hline 48 & N3902 & 6 & N3812 & 1145.0 & 2546 & 36.7 & $9.4(-1)$ & $4.0(-2)$ & $2.2(-1$ \\
\hline 49 & N3929 & 3 & N3883 & 1148.1 & 2108 & 70.5 & $4.6(-1)$ & $2.1(-2)$ & $6.6(-2$ \\
\hline 50 & N3906 & 13 & N3726 & 1148.2 & 4945 & 9.5 & $1.3(+1)$ & $3.4(-1)$ & 2.6 \\
\hline 51 & U06923 & 9 & N3992 & 1154.2 & 5323 & 10.8 & 4.4 & $2.8(-1)$ & 1.9 \\
\hline 52 & U06925 & 10 & N3995 & 115 & 3228 & 31.5 & $3.3(-1)$ & $3.1(-2)$ & $1.9(-1)$ \\
\hline 3 & N4005 & 4 & & 1155 & 2526 & 44.2 & $5.5(-1)$ & $9.6(-3)$ & $4.4(-2$ \\
\hline 44 & N4004 & 3 & N4017 & 1155.7 & 2807 & 34.6 & $9.2(-1)$ & $5.0(-2)$ & $1.8(-$ \\
\hline 55 & N4026 & 3 & & 1156.5 & 5210 & 8.7 & $2.6(+$ & $3.4(-1)$ & 3.7 \\
\hline 56 & N3949 & 18 & N3938 & 1159.1 & 4649 & 7.6 & 6.6 & $4.6(-1)$ & 2.6 \\
\hline 57 & N4061 & 4 & N4070 & 1202.0 & 2041 & 73.2 & 1.0 & $1.0(-3)$ & $1.5(-2$ \\
\hline 58 & N4128 & 3 & & 1203.7 & 6930 & 23.2 & $7.8(-1)$ & $4.0(-2)$ & $1.7(-$ \\
\hline 59 & N4117 & 6 & N4151 & 120 & 4156 & 9.8 & 6.6 & $3.8(-1)$ & 1.6 \\
\hline 60 & N4116 & 3 & N4123 & 1207.0 & 234 & 13.0 & 3.0 & $2.9(-1)$ & $6.1(-1$ \\
\hline 61 & N4205 & 4 & N4036 & $120 \mathrm{~s}$ & 6359 & 14.3 & 1.8 & $1.8(-1)$ & $8.0(-1$ \\
\hline 62 & N4189 & 3 & & 1209.8 & 1408 & 21.6 & 1.3 & $1.2(-1)$ & $2.8(-1$ \\
\hline 63 & N4185 & 8 & N4169 & 1210.1 & 2922 & 38.8 & $4.7(-1$ & $2.9(-2)$ & $3.4(-$ \\
\hline 64 & N4081 & 3 & N4125 & 1210.2 & 6508 & 13.3 & 5.7 & $1.7(-1)$ & 1.2 \\
\hline 65 & N4197 & 4 & N4215 & 1212.6 & 601 & 20.9 & 2.4 & $1.4(-1)$ & $6.7(-1$ \\
\hline 66 & U07395 & 3 & $\mathrm{~N} 4229$ & 1212.9 & 3223 & 67.6 & $1.2(-1)$ & $1.0(-2)$ & $2.2(-2$ \\
\hline 67 & N4256 & 5 & & 1213.8 & $66 \quad 19$ & 25.4 & $7.3(-1)$ & $5.5(-2)$ & $2.0(-1$ \\
\hline 68 & $\mathrm{~N} 4200$ & 3 & N4168 & 1214.5 & 1248 & 23.2 & 2.2 & $1.0(-1)$ & $3.3(-$ \\
\hline 69 & N4235 & 8 & N4378 & 1216 & 644 & 25.9 & 1.4 & 7.8( & $5.2(-$ \\
\hline 70 & I3115 & 9 & $\mathrm{~N} 4261$ & 121 & 609 & 22.8 & 1. & $1.2(-1)$ & $6.4(-1$ \\
\hline 71 & & 3 & & 121 & 1342 & 24.8 & 1.3 & & $2.6(-$ \\
\hline 72 & $\mathrm{~N} 4251$ & 3 & N4314 & 1217 & 2924 & 10.1 & $1.1(+1)$ & $5.1(-1)$ & $9.6(-$ \\
\hline 73 & N4303 & 3 & & 1218.5 & 433 & 15.8 & $1.2(+2)$ & $2.1(-1)$ & $7.8(-$ \\
\hline 74 & $\mathrm{~N} 4290$ & 3 & & 1218.5 & 5824 & 30.8 & $6.0(-1)$ & $3.8(-2)$ & $1.0(-$ \\
\hline 75 & N4306 & 3 & N4313 & 1220.1 & 1216 & 15.1 & 3.3 & $2.5(-1)$ & $2.5(-$ \\
\hline 76 & N4274 & 4 & & 1220.7 & 3024 & 9.0 & $1.5(+1)$ & $5.5(-1)$ & 2.3 \\
\hline 77 & N4510 & 5 & N4545 & 1222.1 & 6456 & 27.8 & $8.5(-1)$ & $5.3(-2)$ & $2.7(-$ \\
\hline 78 & N4335 & 4 & & 1222.4 & 5750 & 46.4 & $2.4(-1)$ & $1.3(-2)$ & $3.9(-$ \\
\hline
\end{tabular}


TABLE 1(continued)

\begin{tabular}{|c|c|c|c|c|c|c|c|c|c|}
\hline & Center & $n$ & Brightest & $\alpha$ & $\delta$ & $\mathrm{D}$ & $\rho$ & $\rho^{*}$ & $\rho_{g}$ \\
\hline 79 & $\mathrm{~N} 4472$ & 7 & & 1223.4 & 807 & 10.2 & 7.7 & $4.2(-1)$ & 4.8 \\
\hline 80 & N4340 & 14 & N4293 & 1224.0 & 1503 & 9.4 & 6.1 & $5.0(-1)$ & 3.1 \\
\hline 81 & U07428 & 3 & N4525 & 1224.2 & 3135 & 12.1 & 5.7 & $3.7(-1)$ & $5.5(-1)$ \\
\hline 82 & N4412 & 6 & N4527 & 1224.4 & 408 & 17.1 & 4.8 & $1.6(-1)$ & $8.8(-1)$ \\
\hline 83 & N4414 & 3 & & 1224.4 & 2925 & 7.2 & $1.1(+1)$ & $5.4(-1)$ & 2.8 \\
\hline 84 & N4461 & 4 & & 1224.6 & 1302 & 19.2 & 4.4 & $1.6(-1)$ & $5.7(-1)$ \\
\hline 85 & N4308 & 5 & N4631 & 1225.0 & 3104 & 6.2 & $1.9(+1)$ & $5.1(-1)$ & 3.3 \\
\hline 86 & N4519 & 12 & N4486 & 1225.3 & 925 & 12.4 & 4.2 & $3.4(-1)$ & 2.6 \\
\hline 87 & N4421 & 6 & N4321 & 1225.6 & 1509 & 16.1 & 2.8 & $2.0(-1)$ & 1.0 \\
\hline 88 & N4477 & 6 & & 1226.0 & 1318 & 13.7 & 4.0 & $3.2(-1)$ & 1.6 \\
\hline 89 & N4458 & 4 & N4206 & 1226.6 & 1401 & 6.8 & 7.6 & $4.8(-1)$ & 2.5 \\
\hline 90 & I3521 & 9 & N4526 & 1226.7 & 1007 & 5.6 & 6.8 & $4.2(-1)$ & 1.3 \\
\hline 91 & N4350 & 4 & & 1226.7 & 1716 & 12.7 & 4.4 & 3.7 & 1.7 \\
\hline 92 & N4291 & 8 & & 1227.8 & 7440 & 17.0 & $5.1(-$ & $5.0(-2)$ & $3.0(-1)$ \\
\hline 93 & $\mathrm{~N} 4435$ & 4 & $\mathrm{~N} 4382$ & 1229.2 & 1535 & 7.9 & 7.6 & $4.7(-1)$ & 1.4 \\
\hline 94 & N4501 & 3 & & 1229.4 & 1441 & 22.8 & 1.4 & $1.1(-1)$ & $4.7(-1)$ \\
\hline 95 & $1226+43$ & 14 & N5055 & 1230.0 & 4411 & 5.3 & 5.4 & $4.8(-1)$ & 5.1 \\
\hline 96 & N4568 & 4 & & 1233.6 & 1035 & 22.5 & 1.9 & $1.2(-1)$ & $4.1(-1)$ \\
\hline 97 & $\mathrm{~N} 4623$ & 3 & N4570 & 1234.1 & 756 & 17.7 & 3.5 & $1.5(-1)$ & $5.4(-1)$ \\
\hline 98 & N4587 & 3 & N4636 & 1234.2 & 315 & 9.1 & $1.7(+1)$ & $3.6(-1)$ & 1.2 \\
\hline 99 & N4596 & 3 & & 1234.6 & 1039 & 18.5 & 2.1 & $1.7(-1)$ & $9.0(-1)$ \\
\hline 00 & N4564 & 8 & N4649 & 1234.9 & 1124 & 11. & $1.1(+1)$ & $4.0(-1)$ & 2.8 \\
\hline 01 & N4598 & 4 & N4535 & 1235.1 & 758 & 19. & 2.0 & $1.6(-1)$ & $5.8(-1)$ \\
\hline .0 & N4600 & 10 & N4665 & 1237.2 & 503 & 7. & 5.4 & $3.7(-1)$ & 2.3 \\
\hline 103 & I3651 & 3 & N4615 & 1238.8 & 2633 & 47. & 3.3 & $9.6(-3)$ & $2.7(-2)$ \\
\hline 100 & N4687 & 3 & U07812 & 1242.6 & 3432 & 42. & $6.7(-2)$ & $5.7(-3)$ & $5.2(-3)$ \\
\hline 0 & $\mathrm{~N} 4725$ & 3 & & 1243.7 & 2602 & 12. & 5.7 & $3.3(-1)$ & $7.2(-1)$ \\
\hline 06 & N4695 & 4 & N4686 & 1245.0 & 5439 & 49.4 & $9.0(-1)$ & $1.7(-2)$ & $2.7(-2)$ \\
\hline 107 & N4762 & 3 & N4654 & 1245.9 & 1113 & 10.2 & $1.1(+1)$ & $4.0(-1)$ & 2.1 \\
\hline 108 & N4795 & 3 & & 1252.1 & 853 & 28.0 & 2.6 & $2.3(-2)$ & $4.3(-2)$ \\
\hline 109 & N48 & 4 & N4819 & 1254.0 & 2757 & 68.2 & $2.8(-1)$ & $2.5(-2)$ & $6.8(-2)$ \\
\hline 10 & N49 & 3 & N4845 & 1254.9 & 113 & 11.8 & 4.3 & $2.5(-1)$ & $4.1(-1)$ \\
\hline 11 & N4853 & 3 & N4839 & 1255.2 & 2742 & 75.5 & $5.4(-1)$ & $1.7(-2)$ & $6.9(-2)$ \\
\hline 12 & I4189 & 5 & N4914 & 1301.5 & 3628 & 47.4 & $4.4(-1)$ & $1.2(-2)$ & $1.0(-2)$ \\
\hline 13 & N4966 & 3 & N4944 & 1304.7 & 2914 & 69.9 & $2.6(-1)$ & $1.9(-2)$ & $3.5(-2)$ \\
\hline 14 & N5056 & 3 & & 1312.1 & 3034 & 56.0 & $1.5(-1)$ & $1.2(-2)$ & $2.0(-2)$ \\
\hline 115 & N5112 & 3 & & 1315.8 & 3822 & 9.8 & 6.5 & 3.3 $(-1)$ & $7.6(-1)$ \\
\hline 116 & N5218 & 3 & & 1322.6 & 6251 & 28.9 & $8.6(-1)$ & $3.8(-2)$ & $6.4(-2)$ \\
\hline 117 & N5142 & 3 & N5149 & 1323.1 & 3630 & 53.6 & $9.0(-2)$ & $5.7(-3)$ & $5.8(-3)$ \\
\hline
\end{tabular}


TABLE 1(continued)

\begin{tabular}{|c|c|c|c|c|c|c|c|c|c|}
\hline & Center & $n$ & Brightest & $\alpha$ & $\delta$ & $\mathrm{D}$ & $\rho$ & $\rho^{*}$ & $\rho_{g}$ \\
\hline 118 & N5208 & 3 & I 900 & 1330.8 & 812 & 68.0 & $1.5(-1)$ & $1.3(-2)$ & $1.8(-2)$ \\
\hline 119 & U08561 & 3 & & 1332.0 & 3411 & 72.9 & $4.2(-1)$ & $1.2(-2)$ & $3.2(-2)$ \\
\hline 120 & N5221 & 3 & N5230 & 1332.7 & 1400 & 69.8 & $7.6(-1)$ & $1.7(-2)$ & $3.6(-2)$ \\
\hline 121 & N5320 & 7 & N5371 & 1347.3 & 4054 & 25.9 & 1.1 & $5.7(-2)$ & $3.5(-1)$ \\
\hline 22 & N5379 & 5 & N5322 & 1347.5 & 6025 & 17.8 & 1.0 & $7.8(-2)$ & $2.0(-1)$ \\
\hline 23 & N5300 & 3 & N5364 & 1351.0 & 459 & 11.9 & 2.3 & $2.1(-1)$ & $4.7(-1)$ \\
\hline 24 & I 948 & 3 & & 1351.1 & 1409 & 68.8 & 1.2 & $9.6(-3)$ & $1.6(-2)$ \\
\hline 25 & N5383 & 7 & & 1351.8 & 4115 & 22.6 & $9.9(-1)$ & $8.0(-2)$ & $4.6(-1)$ \\
\hline 26 & N5341 & 4 & N5395 & 1353.6 & 3754 & 35.6 & $2.8(-1)$ & $2.3(-2)$ & $4.8(-2)$ \\
\hline 27 & U08883 & 3 & U08827 & 1354.2 & 1530 & 56.1 & $5.7(-1)$ & $1.5(-2)$ & $3.2(-2)$ \\
\hline 128 & N5382 & 3 & N5374 & 1355.5 & 629 & 43.0 & $9.0(+1)$ & $1.5(-2)$ & $4.1(-2)$ \\
\hline 129 & N5416 & 3 & & 00.0 & 942 & 61.7 & $2.4(-1)$ & $1.2(-2)$ & $3.2(-2)$ \\
\hline 130 & N5434 & 3 & N5417 & 1400.1 & 838 & 46.6 & $2.0(-1)$ & $1.5(-2)$ & $1.5(-2)$ \\
\hline 31 & N5445 & 4 & N5444 & 1401.2 & 3524 & 38.6 & $5.5(-1)$ & $2.9(-2)$ & $9.5(-2)$ \\
\hline 32 & U09081 & 3 & N5406 & 1402.1 & 39.28 & 52.9 & $1.8(-1)$ & $1.2(-2)$ & $1.6(-2)$ \\
\hline 133 & U09056 & 7 & N5448 & 1405.1 & 4949 & 20.0 & $9.9(-1)$ & $8.8(-2)$ & $7.9(-1)$ \\
\hline 134 & U08980 & 3 & U08975 & 1405.7 & 3916 & 58.0 & $1.2(-1)$ & $1.2(-2)$ & $1.9(-2)$ \\
\hline 135 & U09062 & 3 & N5490 & 1408.7 & 1901 & 50.4 & $1.8(-1)$ & $1.2(-2)$ & $2.3(-2)$ \\
\hline 136 & N5631 & 8 & N5678 & 1413.1 & 5648 & 19.7 & $9.3(-1)$ & $8.0(-2)$ & $4.9(-1)$ \\
\hline 137 & I1000 & 3 & & 1420 & 1807 & 56.6 & $2.2(-1)$ & $1.2(-2)$ & $2.2(-2)$ \\
\hline 138 & N55 & 5 & N5557 & 1420.8 & 3606 & 32.5 & $3.7(-1)$ & $3.2(-2)$ & $1.4(-1)$ \\
\hline 139 & U09 & 6 & N5665 & 25.4 & 1232 & 22.7 & $2.7(-1)$ & $1.7(-2)$ & $8.6(-2)$ \\
\hline 140 & N5641 & 3 & & 1426.8 & 2859 & 43.5 & $6.4(-1)$ & $1.3(-2)$ & $5.1(-2)$ \\
\hline 141 & N5649 & 3 & & 1427.7 & 1410 & 51.9 & 1.2 & $1.2(-2)$ & $4.8(-2)$ \\
\hline 142 & N5653 & 3 & & 1427.8 & 3216 & 35.1 & 1.1 & $2.7(-2)$ & $6.1(-2)$ \\
\hline 143 & N5669 & 3 & & 1428.1 & 1032 & 13.5 & $8.9(-1)$ & $8.6(-2)$ & $1.1(-1)$ \\
\hline 144 & N5689 & 7 & N5676 & 1431.6 & 4934 & 21.8 & 1.0 & $8.4(-2)$ & $4.4(-1)$ \\
\hline 145 & N5675 & 3 & N5695 & 1433.2 & 3641 & 41.7 & $5.7(-1)$ & $1.9(-2)$ & $2.8(-2)$ \\
\hline 146 & N5735 & 3 & & 1435.1 & 2940 & 37.8 & $1.8(-1)$ & $1.3(-2)$ & $3.2(-2)$ \\
\hline 147 & N5692 & 19 & N5566 & 1436.1 & 335 & 15.9 & 1.0 & $8.0(-2)$ & $6.0(-1)$ \\
\hline 148 & & 4 & N5798 & 144 & 3206 & 17.5 & $2.7(-1)$ & $2.3(-2)$ & $3.1(-2)$ \\
\hline 149 & N58 & 4 & N5797 & 145 & 4958 & 40.9 & $9.3(-1)$ & $1.2(-2)$ & 3.3 $(-1)$ \\
\hline 150 & N58 & 3 & & 1501.3 & 204 & 13.9 & 4.8 & $7.8(-2)$ & $3.3(-1)$ \\
\hline 151 & N5820 & 6 & N5905 & 1503.9 & 5446 & 32.3 & $2.0(-1)$ & $1.9(-2)$ & $5.1(-2)$ \\
\hline 152 & N5906 & 3 & N5866 & $15 \quad 17.3$ & 5624 & 6.6 & $4.1(+1)$ & $2.0(-1)$ & $6.1(-1)$ \\
\hline 153 & N5930 & 4 & N5899 & 1521.7 & 4140 & 25.8 & 1.7 & $1.3(-2)$ & $4.6(-2)$ \\
\hline 154 & N5985 & 3 & & 1528.7 & 5941 & 25.4 & $4.7-1$ & $1.9(-2)$ & $2.3(-2)$ \\
\hline 155 & I4564 & 6 & 14567 & 1532.3 & 4327 & 56.7 & $1.8(-1)$ & $1.5(-2)$ & $1.7(-2)$ \\
\hline 156 & N5974 & 3 & N5958 & 1534.3 & 3035 & 19.5 & $3.6(-1)$ & $1.5(-2)$ & $3.0(-2)$ \\
\hline
\end{tabular}


TABLE 1(continued)

$\begin{array}{lllllccccc} & \text { Center } & n & \text { Brightest } & \alpha & \delta & \mathrm{D} & \rho & \rho^{*} & \rho_{g} \\ 157 & \text { N5956 } & 8 & \text { N5962 } & 1535.5 & 1414 & 419.1 & .5 .2(-1) & 2.8(-2) & 8.1(-2) \\ 158 & \text { N5982 } & 3 & & 1539.0 & 5913 & 29.3 & 1.6 & 1.9(-2) & 4.2(-2) \\ 159 & \text { I1151 } & 3 & & 1550.7 & 1821 & 21.5 & 3.8(-1) & 2.3(-2) & 6.8(-2) \\ 160 & \text { U10127 } & 3 & \text { N6052 } & 1557.3 & 2059 & 48.0 & 2.1(-1) & 1.9(-2) & 4.2(-2) \\ 161 & \text { I1174 } & 3 & \text { N6021 } & 1602.1 & 1602 & 47.3 & 3.0(-1) & 1.5(-2) & 4.0(-2)\end{array}$

There are many coincidences between groups from Table 1 and groups from other catalogs (Turner and Gott 1976, Huchra and Geller 1982, Vennik 1984, Tully 1987). Some groups from the Geller and Huchra (1983) catalog are merged in Table 1 and vice versa, some groups from Table 1 are merged in the GH catalog.

We thank D. Stefanova who helped in the preparation of the catalog. This work is supported in part by the Bulgarian Committee for Science (contract 943).

\section{REFERENCES}

Geller, M. J., and Huchra, J. P. 1983, Ap.J.Suppl., 52, 61.

Huchra, J., Davis, M., Latham, D., and Tonry, J. 1983, Ap.J.Suppl., 52, 89.

Huchra, J. P., and Geller, M. J. 1982, Ap.J., 257, 423.

Kalinkov, M., and Kuneva, I. 1985, Astr.Nach., 306, 283.

-. 1986, M.N.R.A.S., 218, 49p.

Tully, R. B. 1987, Ap.J., 321, 280.

Turner, E. L., and Gott, J. R. 1976, Ap.J.Suppl., 32, 409.

Vennik, J. 1984, Tartu Astr.Obs. Teated, No. 73, p. 3. 